\title{
The effects of high gravitational stress on the expression of stress response genes in Arabidopsis thaliana
}

\author{
Sophia Zappia ${ }^{1}$, Richard Barker ${ }^{2}$, and Simon Gilroy ${ }^{2}$ \\ ${ }^{I}$ Student, ${ }^{2}$ Professor (Botany, University of Wisconsin-Madison) Madison, Wisconsin, USA
}

\begin{abstract}
As humans venture farther into space, we are increasingly interested in how plants respond to the unique conditions of spaceflight. This experiment focuses on how gene expression in the model plant Arabidopsis thaliana is affected by high-gravitational stress that is comparable to the amount experienced on a sounding rocket. The genes chosen, have been studied previously for their roles in mechanical stress and pathogen response. Our results showed an increase in expression of the genes TCH2, TCH3, and MPK3, no change in the expression of CBP60g, and a decrease in the expression of RCI3 and ICS1. These results are largely consistent with spaceflight data and warrant further investigation into the effects of rocket launch on plant growth.
\end{abstract}

\section{Background}

Signaling mechanisms in plants are highly complex and responsive to a variety of stimuli. This experiment focuses on three groups of genes involved in plant stress - Calcium signaling, peroxidases, and pathogen response genes. The genes are involved in response to a variety of both abiotic and biotic stressors.

Table 1. A brief description of each gene in this study.

\begin{tabular}{|l|l|l|l|l|}
\hline Gene Name & Abbreviation & AT locus & Category & Brief Description \\
\hline Touch 2 & TCH2 & AT5G37770 & Ca2+ signaling & Involved in flooding response and mechanical stress. \\
\hline Touch 3 & TCH3 & AT2G41100 & Ca2+ signaling & Involved in mechanical, cold, and light stress. \\
\hline Cam-binding protein 60-like G & CBP60g & AT5G26920 & $\begin{array}{l}\text { Ca2+ signaling, } \\
\text { Pathogen Response }\end{array}$ & $\begin{array}{l}\text { Triggered by a variety of abotic and biotic stressors. } \\
\text { Recruited to the promoter of ISC1. }\end{array}$ \\
\hline Isochorismate synthase 1 & MPK3 & AT3G45640 & Pathogen Response & $\begin{array}{l}\text { Increases in response to touch, cold, and fungus } \\
\text { attack. }\end{array}$ \\
\hline Rare cold inducible 3 & ICS1 & AT1G74710 & Pathogen Response & Involved in Salicylic Acid signaling. \\
\hline Peroxidase 22 & & AT1G05260 & Peroxidase & $\begin{array}{l}\text { Peroxidase activity gene that is induced by cold and } \\
\text { desiccation. }\end{array}$ \\
\hline
\end{tabular}

Using data from the STRING database (Snel B. et al., 2000), we generated a map of the connections in molecular pathways of these genes. Note that CBP60g binds to the promoter of ICS1. 


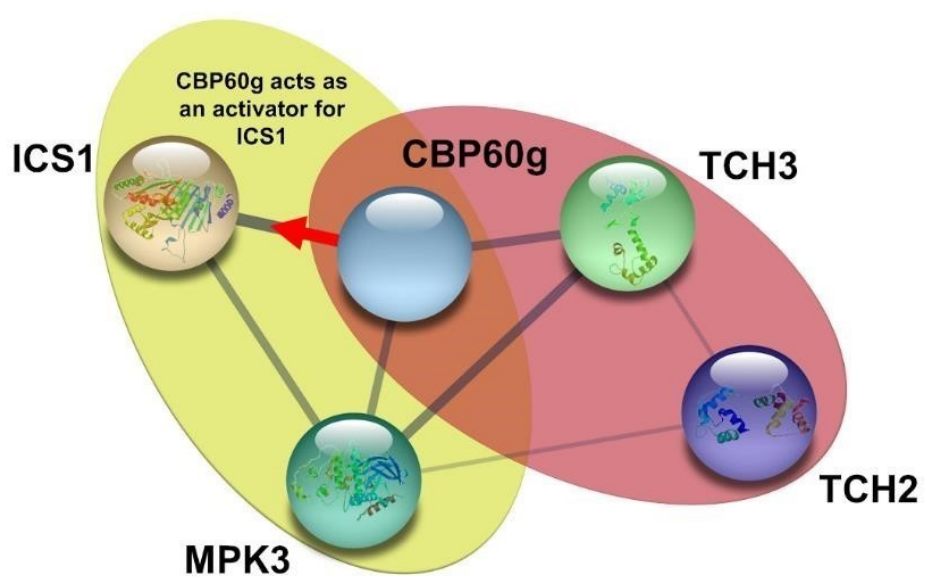

MPK3

\section{PRX22}

$\mathrm{RCl} 3$

\section{Calcium Signaling \\ Peroxidase \\ Pathogen Response}

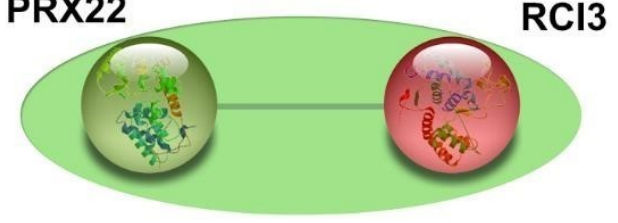

Fig. 1. A map of relatedness between the genes studied. A thicker line indicates a closer connection.

Many of the genes have been shown to be highly correlated in previous research. RNA coexpression stores from the STRING database are shown in Table 2, where a higher co-expression score indicates that the genes have been affected in similar ways in previous experiments.

Table 2. RNA co-expression scores based on previous research. Data from the STRING database (Snel B. et al. 2000).

\begin{tabular}{|l|r|r|r|r|r|r|r|}
\cline { 2 - 8 } \multicolumn{1}{l|}{} & \multicolumn{1}{l|}{$\mathrm{RCI3}$} & $\mathrm{PRX} 22$ & $\mathrm{TCH} 2$ & $\mathrm{TCH} 3$ & $\mathrm{CBP} 6 \mathrm{~g}$ & $\mathrm{MPK} 3$ & \multicolumn{1}{l|}{$\mathrm{ICS} 1$} \\
\hline $\mathrm{RCl} 3$ & & 0.424 & & & & & \\
\hline $\mathrm{PRX} 22$ & 0.424 & & & & & & \\
\hline $\mathrm{TCH} 2$ & & & & 0.177 & & 0.180 & \\
\hline $\mathrm{TCH} 3$ & & & 0.177 & & 0.438 & 0.471 & \\
\hline $\mathrm{CBP} 60 \mathrm{~g}$ & & & & 0.438 & & 0.418 & 0.168 \\
\hline $\mathrm{MPK3}$ & & & 0.180 & 0.471 & 0.418 & & 0.054 \\
\hline $\mathrm{ICS} 1$ & & & & & 0.168 & 0.054 & \\
\hline
\end{tabular}

PRX22 and RCI3, the two peroxidases, are strongly correlated in expression levels. TCH3, CBP60g, and MPK3 are strongly correlated with each other. TCH2 is moderately linked with TCH3 and MPK3; ICS1 is moderately linked to CBP60g and weakly to MPK3. 


\section{Methods}

Wild-type Arabidopsis thaliana seedlings were grown with 6 seeds each in 24 small pots using Sungro Fafard ${ }^{\circledR}$ Germinating Mix, which provided all necessary nutrients. They were grown under $24 \mathrm{hr}$ light for 11 days and watered every three days. The pots were divided evenly in an alternating fashion into treatment and control groups. The treatment group was loaded into the centrifuge, where they received $12 \pm 1$ Gs of force, which is comparable to the maximum force of $12 \mathrm{Gs}$ experienced on sounding rockets (European Space Agency, n.d) They were spun for a duration of 30 seconds, then all plants were flash frozen in liquid nitrogen.

RNA was extracted from the roots and shoots of the plants using the protocol described by Choi et. Al (2018), and RNA concentration, OD260/280 ratios and OD260/230 ratios were obtained using a nanodrop machine. The four samples from each group with the highest concentration and purity underwent further analysis.

Reverse Transcription-QPCR was used, with Ubiquitin 10 as a housekeeping gene. Three QPCR plates were used, which contained 96 wells each. Each well contained 24 ng of RNA, and each row contained a primer for the target gene. Each reaction well was run with four technical replications per gene per biological replicate.

The plates were ran using a 7500 Real-Time PCR System. The thermocycler went through a warming step then forty PCR cycle steps. At each step, the fluorescence level was measured, and the cycle number was recorded when fluorescence reached the threshold level, giving the $\mathrm{C}_{\mathrm{T}}$ (Cycle threshold) value.

Fold change values of each gene were calculated using the $2^{-\Delta \Delta \mathrm{C}}$ method. The Cycle $C_{\mathrm{T}}$ value was obtained for each of the 96 wells on the three plates. Using Python and Jupyter, the $\Delta \mathrm{CT}_{\mathrm{T}}$ values for each target gene by sample were calculated by subtracting the control gene Ubiquitin $10 \mathrm{C}$ values from the target gene $\mathrm{C}_{\text {r. The control }} \Delta \mathrm{C}_{\mathrm{T}}$ values for each gene were then subtracted from the treatment $\Delta \mathrm{C}$ T values to give the $\Delta \Delta \mathrm{C}$ T value. Taking $2^{-\Delta \Delta \mathrm{C}_{\mathrm{r}}}$ gave the fold change value.

\section{Results}

The results showed an increase in the expression of TCH3, MPK3, and TCH2. There was no significant change in the expression of CBP60g, and there was an extremely significant decrease in RCI3, and a fairly significant decrease in ICS1. PRX22 had overall low expression levels and was excluded from analysis due to low signal-to-noise ratios. A heatmap of expression $\left(\Delta \mathrm{CT}_{\mathrm{T}}\right)$ is in Fig. 2. Red indicates a lower $\Delta \mathrm{CT}_{\mathrm{T}}$ value, indicating higher expression values, and blue higher $\Delta \mathrm{C}_{\mathrm{T}}$ value (lower expression). 


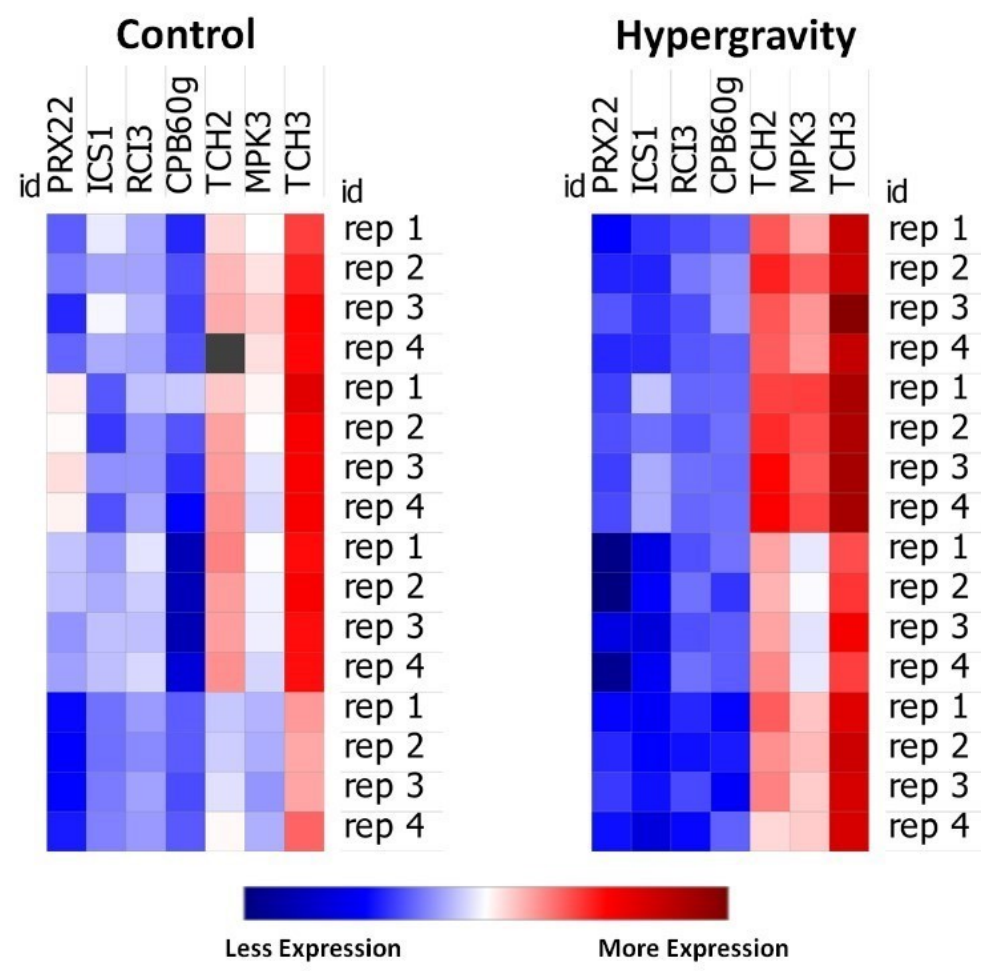

Fig. 2. A heatmap of expression levels in the two treatment groups. Created using Morpheus.

The values were graphed A T-test was performed, and P values were obtained for each of the genes and can be seen in Table 3. A graph of fold change values is shown in Fig. 3. A value greater than one indicates gene activation, and a level less than one indicates gene suppression.

Gene Expression After Short-term High G-force

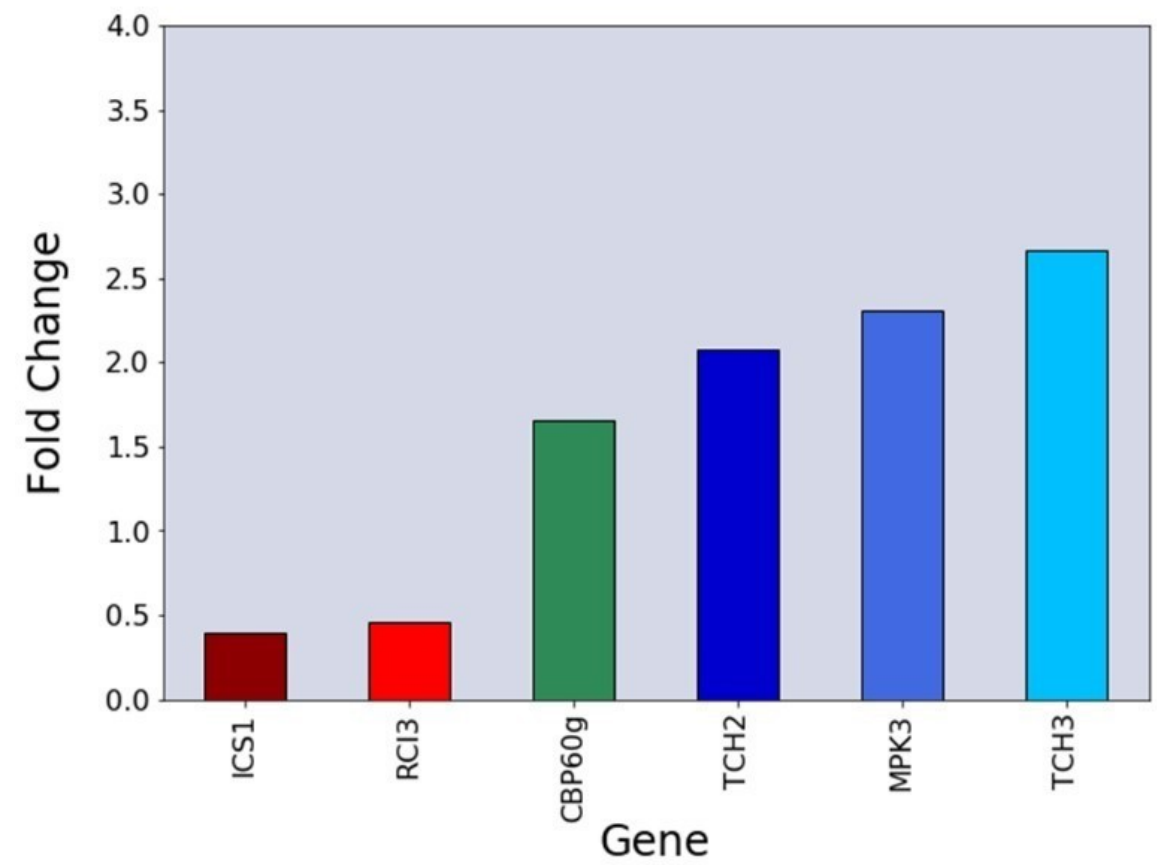

Fig. 3. A bar chart showing the fold change values for each gene, where 1.0 is baseline expression. 
Table 3. A table of fold change values and $\mathrm{P}$ values for each gene studied.

\begin{tabular}{|l|r|r|r|r|r|r|}
\hline Gene & \multicolumn{1}{|c|}{ TCH3 } & \multicolumn{1}{l|}{ MPK3 } & \multicolumn{1}{l|}{ TCH2 } & \multicolumn{1}{l|}{ CBP60g } & \multicolumn{1}{l|}{ RCl3 } & ICS1 \\
\hline Fold Change & 2.587 & 2.255 & 2.158 & 0.950 & 0.506 & 0.409 \\
\hline Significance & 0.0792 & 0.0706 & 0.0805 & 0.3442 & 0.0027 & 0.0654 \\
\hline
\end{tabular}

The results indicate that the genes involved in mechanical stress (TCH2, TCH3, MPK3) are significantly increased when compared to plants that underwent similar handling, but not centrifugation.

RCI3 sharply decreased, which may indicate that peroxidase activity, which is known to be reduced during spaceflight (Kwon T. et al., 2015), may also be affected by the mechanical stress of the rocket takeoff. Both are of major concern for plants launched on commercial spaceflight launches as mechanical stress, and a reduced capacity to withstand oxidative stress can severely affect the growth and yield of crop plants.

\section{Further Research}

Agravitropic mutants such as arg1 and toc132 lack a gravitropic response. They would be used to determine whether gravitropic sensing, specifically through statolith granules, are responsible for the change in gene expression during rocket launch.

Lanthanum chloride can be used to chemically suppress gravitropic and other stress responses (Friedman et al., 1998) and potentially numb the plants to gravitational stress during rocket launch. This would allow us to test whether the gravitational stress can be mitigated by chemical agents.

The duration of force experienced may show different effects, particularly in genes later in the stress response pathway such as CBP60g. As this experiment shows only the immediate responses, taking samples at specific intervals after launch will allow us to see into the long-term mechanisms of gravitational stresses. In addition, the centrifuge is currently being upgraded to support exact thrust curve simulations of commercial rockets such as Blue Origin's New Shepard and the SpaceX Falcon 9.

Another method for quantifying the effect of gravitational stress is using G-CAMP, a "high affinity $\mathrm{Ca}^{2+}$ probe composed of a single GFP" (Nakai et al., 2001). This technology allows us to see calcium signal propagation in living cells, giving a dynamic view into calcium signaling responses.

\section{Acknowledgments}

This project is funded by the Wisconsin Space Grant Consortium's Undergraduate Research Fellowship. Special thanks to Dr. Richard Barker, Dr. Arthur Poitout, Wilhelm Kessenich, Dr. Arkadipta Bakshi, Dr. Cullen Vens, Dr. Pavel Pinkas, Madison West Rocket Club, Dr. Sarah Swanson, and Dr. Simon Gilroy. 


\section{References}

Choi, W.-G., R. J. Barker, S.-H. Kim, S. J. Swanson, and S. Gilroy. (2019). Variation in the transcriptome of different ecotypes of Arabidopsis thaliana reveals signatures of oxidative stress in plant responses to spaceflight. American Journal of Botany 106(1): 123-136

European Space Agency. (n.d). Sounding rockets. Retrieved from http://www.esa.int/Science_Exploration/Human_and_Robotic_Exploration/Research/Sounding_ro ckets.

Haya Friedman et al. (1998). Inhibition of the Gravitropic Response of Snapdragon Spikes by the CalciumChannel Blocker Lanthanum chloride. Plant Physiology 118 (2) 483-492;

Krishnakumar, V. et al. (2017). ThaleMine: a warehouse for Arabidopsis data integration and discovery. Plant and Cell Physiology 58(1), pp.e4-e4. DOI: 10.1104/pp.118.2.4

Kwon, T., Sparks, J. A., Nakashima, J., Allen, S. N., Tang, Y., \& Blancaflor, E. B. (2015).

Transcriptional response of Arabidopsis seedlings during spaceflight reveals peroxidase and cell wall remodeling genes associated with root hair development. American Journal of Botany 102(1), 21-35.

Morpheus: Versatile matrix visualization and analysis software. (n.d) Broad Institute. Retrieved from https://software.broadinstitute.org/morpheus

Nakai, J., Ohkura, M., \& Imoto, K. (2001). A high signal-to-noise Ca2+ probe composed of a single green fluorescent protein. Nature Biotechnology, 19(2), 137-141. doi:10.1038/84397

Snel B et al. (2000). STRING: a web-server to retrieve and display the repeatedly occurring neighbourhood of a gene. Nucleic Acids Res. Sep 15;28(18) 